\title{
Evaluación de dos Modelos de Reducción de Escala en la Generación de Escenarios de Cambio Climático en el Valle de Mexicali en México
}

\author{
Adriana Camargo-Bravo* y Rafael O. García-Cueto \\ Instituto de Ingeniería, Universidad Autónoma de Baja California, Blvd. Benito Juárez y \\ Calle de la Normal s/n Col. Insurgentes Este, C.P. 21280, Mexicali, B.C.-México. \\ (e-mail: adriana.camargo@prodigy.net.mx; rafaelcueto@uabc.edu.mx) \\ * Autor a quien debe ser dirigida la correspondencia.
}

Recibido Sep. 08, 2011; Aceptado Nov. 07, 2011; Versión final recibida Nov. 25, 2011

\begin{abstract}
Resumen
Se evaluaron dos modelos de reducción de escala en la generación de escenarios de cambio climático en el Valle de Mexicali en México. Las técnicas comparadas son un Generador Estocástico de Tiempo Meteorológico (LARS-WG) y un método dinámico-estadístico (SDSM). Cada técnica se evaluó por su habilidad para reproducir algunas características estadísticas del clima observado en el período 1961-1990. Se elaboraron escenarios de cambio climático de temperatura y precipitación para los horizontes temporales 2020 y 2050 . El LARS WG y el SDSM son técnicas sencillas que mostraron habilidades diferentes en la simulación de momentos estadísticos del clima observado, siendo ambos más eficientes para simular la temperatura que la precipitación. Para uno de los escenarios considerados se encontró diferencias de $0.1^{\circ} \mathrm{C}$ y $0.2^{\circ} \mathrm{C}$ para temperatura máxima y temperatura mínima y del $40.4 \%$ al $76.7 \%$ para precipitación.
\end{abstract}

Palabras clave: reducción de escala, cambio climático, valle de Mexicali, LARS-WG, SDSM

\section{Evaluation of two Downscaling Models in the Generation of Climate Change Scenarios in Mexicali Valley in Mexico}

\begin{abstract}
Two downscaling models in the generation of climate change scenarios in the Mexicali valley in México were evaluated. The evaluated techniques were the stochastic weather generator (LARSWG) and the dynamical-statistical model (SDSM). Each technique was evaluated for its ability to reproduce some statistical characteristics of the observed climate during the period 1961-1990. Climate change scenarios of temperature and precipitation for the period 2020 to 2050 were considered. LARS WG and SDSM are simple techniques that showed different abilities in simulating statistical moments of the observed climate, but both were more efficient for simulating temperature than precipitation. For one of evaluated scenarios differences of $0.1^{\circ} \mathrm{C}$ and $0.2^{\circ} \mathrm{C}$ for maximum and minimum temperature and $40.4 \%$ to $76.7 \%$ for precipitation.
\end{abstract}

Keywords: downscaling, climate change, Mexicali valley, LARS-WG, SDSM. 


\section{INTRODUCCIÓN}

Los Modelos de Circulación General (MCGs) de la atmósfera usan ecuaciones matemáticas para representar los principales procesos físicos a gran escala, los que posteriormente se enlazan para simular el sistema climático completo (Solomon et al., 2007). Experimentos numéricos con los MCGs permiten concluir que el aumento de las concentraciones atmosféricas de Gases de Efecto Invernadero (GEI), producirá cambios en el clima global y regional, particularmente incrementos en la temperatura y modificación en los patrones de precipitación (Magaña, 2010). Estos cambios pueden simularse con los Escenarios de Emisiones (EE), que se refieren a las diferentes concentraciones de GEI y aerosoles que se utilizan para perturbar el sistema climático y generar estimaciones cuantitativas creíbles, llamadas escenarios del cambio climático futuro. Los EE corresponden a una variedad de hipótesis socioeconómicas consideradas como las fuerzas impulsoras en la producción de las emisiones futuras de GEI (Nakicenovic et al., 2000).

Es menos claro en qué medida el clima en un sitio particular se modificará, debido a que los escenarios climáticos futuros derivados de los MCGs que se presentan en el Cuarto Informe de Evaluación sobre Cambio Climático (CIECC), se refieren a proyecciones de temperatura y precipitación (Solomon et al., 2007); por un lado, con una resolución temporal típica en términos de medias mensuales de las variables climáticas, que no pueden usarse directamente en los modelos de evaluación de impacto en un sitio particular (Semenov, 2007) ya que estos utilizan la entrada de datos climáticos a escala diaria. Por otro lado, su resolución espacial alrededor de los $50,000 \mathrm{~km}^{2}$, no permite considerar los forzantes del clima local como topografía y uso de suelo en sus salidas y los procesos a escala pequeña no pueden ser representados explícitamente (Semenov et al., 2010). Con tal resolución es difícil precisar acciones de adaptación para un sitio o un sector en particular, por lo que las salidas de los MCGs requieren la aplicación de técnicas de reducción de escala (downscaling), las cuales funcionan como puentes entre los resultados obtenidos con los MCGs y los modelos que analizan los potenciales impactos del cambio climático a nivel local. Estas técnicas se clasifican según la forma de regionalización: las dinámicas usan modelos físicos de clima regional y las estadísticas se basan en la regresión lineal, la correlación canónica y las redes neuronales entre otras (Estrada et al., 2010). La base de las técnicas de reducción de escala es que existen relaciones dinámicas o estadísticas entre el clima local y el de gran escala, lo que permite determinar condiciones locales a partir de información de baja resolución; la suposición es que las relaciones estadísticas construidas con datos observados se mantienen válidas aún para condiciones de clima futuro (Semenov et al., 1998; Wilby et al., 2007).

La reducción de escala permite crear escenarios sobre la condición climática más probable, pero cada caso o región es diferente y por ello se requiere analizar la estrategia de reducción de escala más adecuada, pues entre más discontinuidades exhiba la variable a ser escalada, mayor será la dificultad para escalarla, como en el caso de la precipitación. El objetivo de este trabajo es generar a partir de las proyecciones de los MCGs, escenarios de temperatura y precipitación bajo cambio climático, utilizando dos herramientas de escalamiento espacial estructuralmente diferentes, el LARS WG y el SDSM; evaluando previamente su habilidad de reproducir algunas características estadísticas del clima observado 1961-1990, pues de los resultados obtenidos depende la confiabilidad en los cambios proyectados y por lo tanto de la dirección que se tome en las estrategias de mitigación y adaptación. Esta evaluación se realiza para el Valle de Mexicali, situado en el estado de Baja California, una región del noroeste de México afectada por los fenómenos de gran escala y los procesos locales convectivos (Raynal et al., 2004).

\section{METODOLOGÍA}

Área de estudio y base de datos

El Valle de Mexicali se localiza entre los $114^{\circ} 45^{\prime}$ a $115^{\circ} 40^{\prime}$ longitud oeste y $31^{\circ} 40^{\prime}$ a $32^{\circ} 40^{\prime}$ de latitud norte (Figura 1), con una altitud sobre el nivel del mar entre $5 \mathrm{~m}$ y $28 \mathrm{~m}$. Limita al norte con California, Estados Unidos, al sur y sureste con el Golfo de California y la sierra El Mayor, al oeste con la Sierra Cucapá y al este con Arizona, Estados Unidos y la mesa arenosa de San Luis Río Colorado, Sonora. García (1973) denomina al clima de esta región como cálido seco, muy 
extremoso con gran oscilación anual de temperatura y precipitación escasa de régimen intermedio entre verano e invierno. En este valle se ubica la región agrícola formada por la zona de Baja California, municipio de Mexicali y la zona de Sonora, municipio de San Luis río Colorado.

Utilizando el Sistema de Información Geográfica (SIG) ArcGis 10 se elaboró un mapa del área de estudio y con sus coordenadas geográficas se ubicaron las estaciones (Figura 1) que cumplieron el control de calidad aplicado a los datos diarios de temperatura máxima (Tmax), temperatura mínima (Tmin) y precipitación (P) para el periodo base observado 1961-1990. Esta información se obtuvo de la base de datos ERIC III y de la Comisión Nacional del Agua (CNA). Los datos diarios de las variables predictoras, derivados del re-análisis (Kalnay, et al., 1996) del Centro Nacional de Predicción Ambiental (NCEP, por sus siglas en inglés) del período 1961-2001, así como los datos del período 1961-2099 de los EE A2 y B2 derivados de la salida del MCG de baja resolución (2.5 en latitud por $3.75^{\circ}$ en longitud), el HadCM3 (Hadley Center versión 3), se obtuvieron del punto de rejilla geográficamente más próximo a la zona de estudio $\left(Y=22\right.$, latitud: $32.5^{\circ} \mathrm{N}$, y $X=66$, longitud: $\left.116.25^{\circ} \mathrm{W}\right)(\mathrm{CCIS}, 2010)$. En base al punto geográfico más cercano a cada estación, se utilizaron también los datos de los escenarios regionales de cambio climático generados para México por Zermeño (2008) y aplicados por Magaña (2010). Estos son escenarios mensuales de cambios de enero a diciembre de temperatura y precipitación con una resolución espacial de $50 \mathrm{~km}^{2}$, derivados de la combinación de 24 MCGs a la que se denominada Modelo Multiensamble (MM).

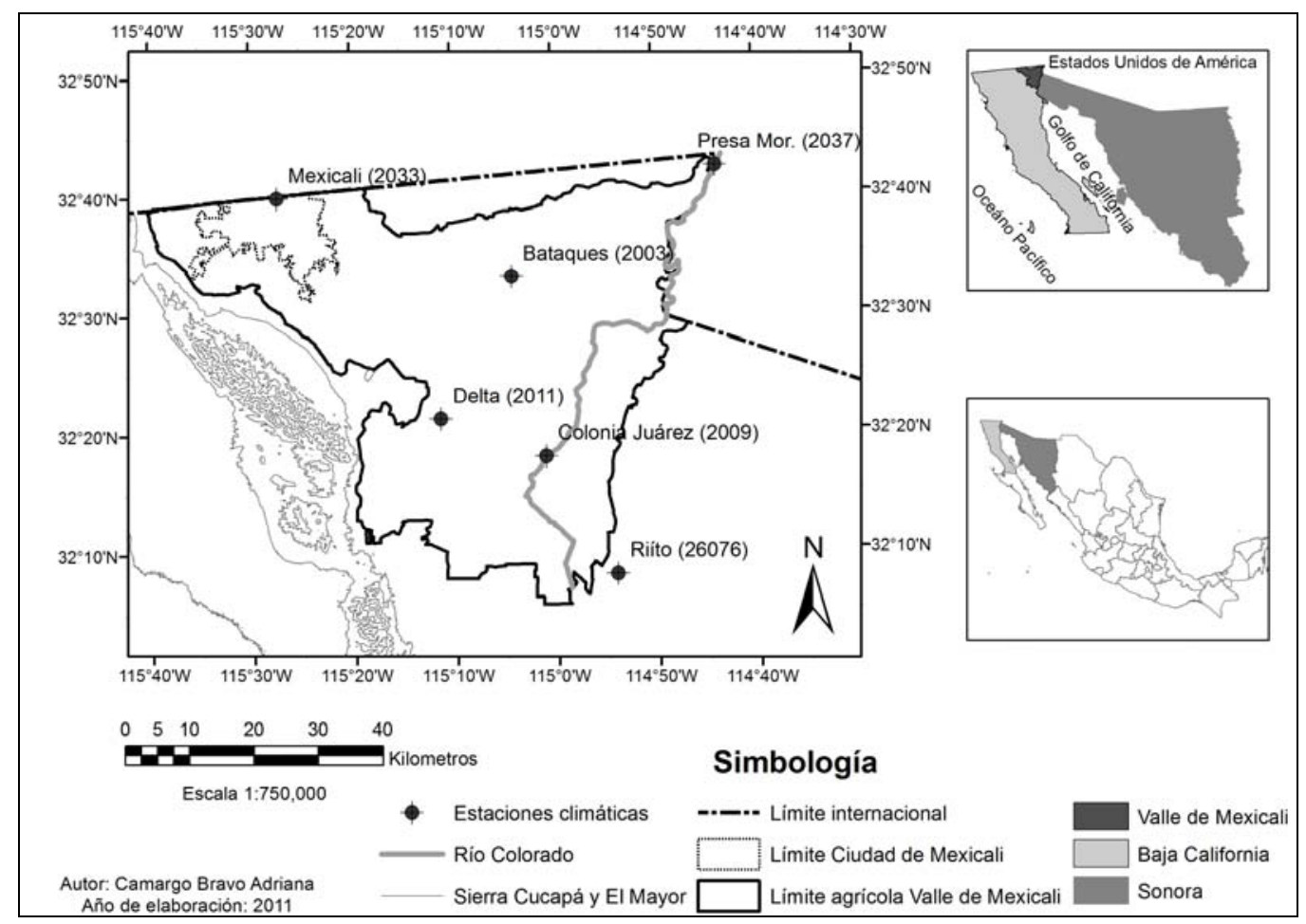

Fig. 1: Mapa de ubicación del Valle de Mexicali y las estaciones climáticas

\section{Modelo SDSM}

EI SDSM (Statistical Downscaling Model, por sus siglas en inglés) es un híbrido entre generación climática estocástica y función de transferencia que se basa en la regresión lineal múltiple, establece relaciones empíricas entre datos diarios observados de temperatura y precipitación principalmente (predictandos a escala local) y predictores de escala regional extraídos de los MCGs, conocidos como variables independientes (Wilby et al., 2007). El SDSM versión 4.2 es un programa de acceso libre (https://co-public.lboro.ac.uk/cocwd/SDSM) y se utilizó como sigue: Primero se analizaron los 26 predictores disponibles en el archivo de datos predictores (NCEP), y se seleccionó para el periodo observado 1961-1990 el subconjunto que mostró el mejor ajuste con el predictando, evaluado a partir del coeficiente de determinación con los valores más altos de correlación inter-variables y los valores de probabilidad (p) más bajos, así como la más alta varianza mensual explicada. 
Después se obtuvo el modelo de regresión calibrando con los datos observados correspondientes al período 1961-1980. Las variables predictoras con las que se correlacionaron en común las 6 estaciones analizadas, fueron la presión media al nivel del mar (mslp) y altura geopotencial a 500 hpa (p500) para la Tmax y la Tmin; y adicionalmente, la Tmax mejoró de forma importante la correlación con la humedad relativa superficial (rhum); mientras que la Tmin con la velocidad zonal a 500 hPa (p5_u) y la humedad específica superficial (shum). Para la precipitación, la humedad específica superficial (shum) fue el predictor común con la que se correlacionaron los 6 sitios de estudio, sumando cierta mejora en la correlación de los sitios, el uso de otras variables predictoras como la velocidad zonal superficial (p_u), vorticidad superficial ( $\left.p_{-} z\right)$, velocidad zonal a $850 \mathrm{hPa}$ (p8_u), vorticidad a $850 \mathrm{hPa}\left(\mathrm{p} 8 \_z\right)$, divergencia a $850 \mathrm{hPa}(\mathrm{p} 8 \mathrm{zh})$, humedad relativa a $500 \mathrm{hPa}$ ( $\mathrm{r} 500)$, humedad relativa a $850 \mathrm{hPa}$ (r850) y humedad relativa superficial (rhum). A nivel anual promedio los mejores resultados para el coeficiente $R^{2}$ se tienen para la temperatura (0.3-0.5), mientras que para la precipitación se muestran valores menores de este coeficiente (0.1-0.2).

Para la temperatura el modelo se definió como no condicional a nivel anual, ya que supone una relación directa entre el predictando y los predictores; mientras que para la precipitación se trabajó con un modelo transformado a la raíz cuarta y se especificó de forma condicional a nivel estacional, debido a la naturaleza bimodal en la región. El modelo se validó con los datos del período observado1961-1990. Finalmente, con un nivel de significancia de 0.05, se generó un ensamble de 20 experimentos de series diarias de los escenarios A2 y B2 derivados del HadCM3, desde el año 1961 hasta el año 2099.

\section{Modelo LARS WG}

EI LARS WG (Long Ashton Research Station Weather Generator, por sus siglas en inglés) genera datos diarios de tiempo de un sitio particular con las mismas características estadísticas de la serie real de la estación (Semenov et al., 1998). Este generador estocástico se basa en series autoregresivas (Semenov, 2007) y utiliza una distribución semi-empirica que se aproxima a una distribución de probabilidad de series húmedas y secas, precipitación diaria, temperatura máxima, temperatura mínima y radiación solar (Semenov et al., 2010). El programa LARS WG versión 5.0 es gratuito (http://www.rothamsted.bbsrc.ac.uk/mas-models/larswg/download.php) y se aplicó de la forma siguiente: primero se calibró el modelo analizando las características estadísticas de los datos observados diarios 1961-1990. Después se validó mediante pruebas estadísticas y se verificó si las series generadas provenían de la misma población. Finalmente se generaron las muestras diarias (100 años) bajo cambio climático a partir de los parámetros estadísticos que se obtuvieron de la calibración y utilizando el archivo de datos de escenarios mensuales según la celda del MM que corresponde a la estación. EI LARS WG construyó las series sintéticas futuras para la climatología 2020 (periodo 2010-2039) y 2050 (periodo 2040-2069) afectadas por los índices del escenario A1B y A2.

\section{RESULTADOS Y DISCUSIÓN}

El periodo base observado 1961-1990 tiene una precipitación media total anual de $70.7 \mathrm{~mm}$ y una temperatura media anual de $21.4{ }^{\circ} \mathrm{C}$ con valores extremos de $-9{ }^{\circ} \mathrm{C}$ y $50{ }^{\circ} \mathrm{C}$. En un análisis de correlación entre datos diarios observados de las 6 estaciones, se presentaron coeficientes de determinación $\left(R^{2}\right)$ de 0.8 a 0.9 para la Tmax y Tmin y para la $P$ de 0.2 a 0.6 . Estos valores mostraron que para la temperatura la zona es más homogénea que respecto a la precipitación.

\section{Validación con el SDSM}

Mediante la herramienta de generador de tiempo, el programa generó 20 series sintéticas a nivel mensual para el periodo1961-1990. Como ejemplo se muestra en la Figura 2 la simulación de la Tmax en la estación Presa Morelos; se puede notar que la reconstrucción a partir de los datos NCEP y del modelo HadCM3 A2, entrega muy buenos resultados para el clima observado 19611990, mientras que el HadCM3 B2 muestra una pequeña pero clara sobreestimación en todo el ciclo anual. La varianza para la Tmax y Tmin es simulada con una correlación entre datos observados y simulados de entre 0.7 y 0.9 , reproduciendo la tendencia del ciclo anual (Figura 3 ), 
pero ofreciendo subestimaciones y sobreestimaciones en diferentes meses en cada uno de los sitios de estudio. Cabe señalar que los niveles de correlación fueron variables entre la simulación con datos del NCEP, el HadCM3 A2 y el HadCM3 B2, siendo la más baja para este último.

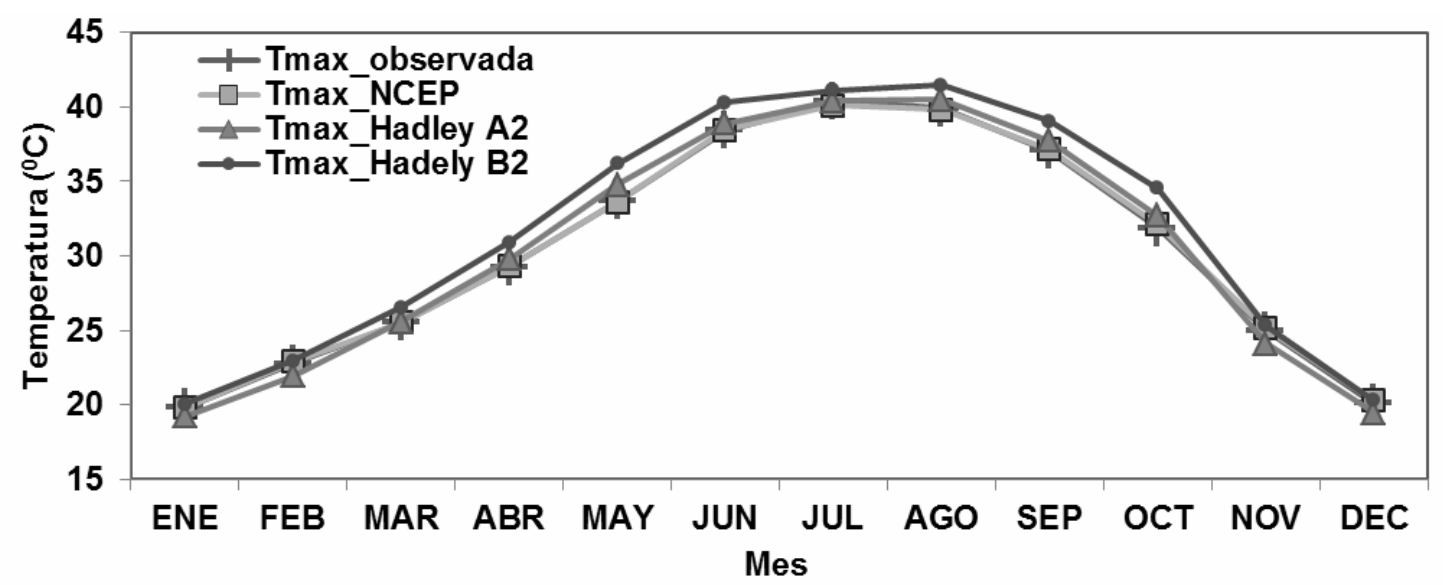

Fig. 2: Simulación de temperatura máxima media mensual del periodo 1961-1990 con observaciones de la estación Presa Morelos usando el modelo SDSM

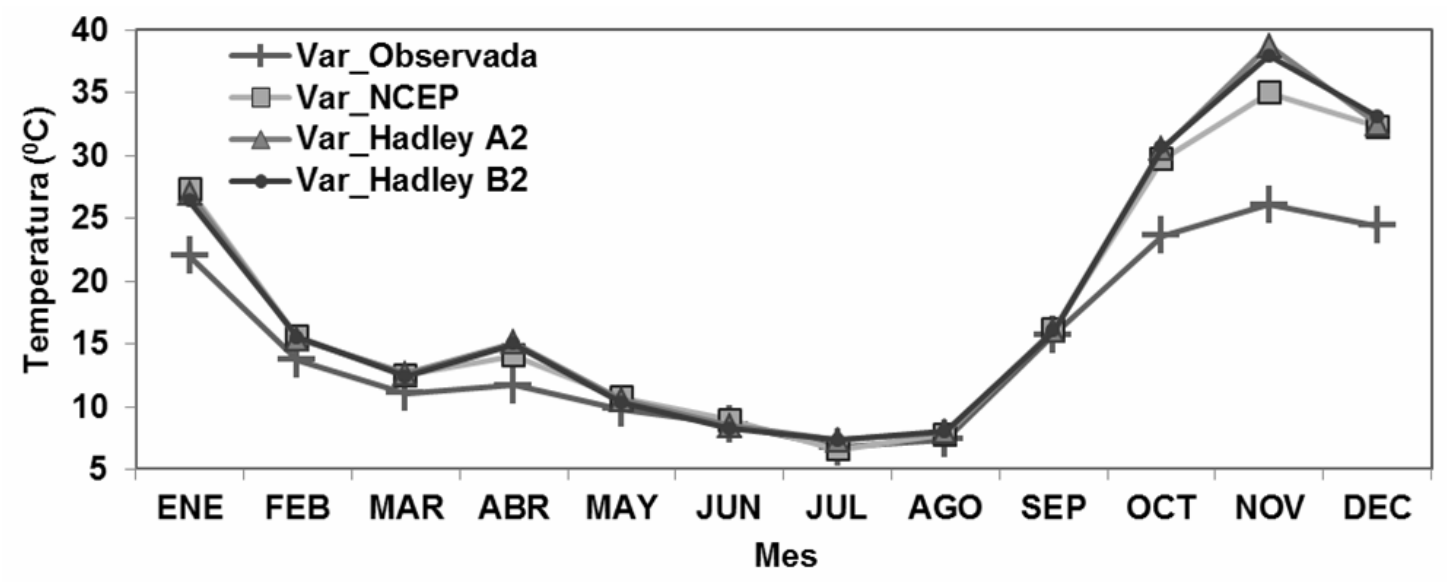

Fig. 3: Simulación de la varianza de la temperatura mínima media mensual del periodo 1961-1990 con observaciones de la estación Bataquez usando el modelo SDSM

Respecto a la precipitación, la reconstrucción del ciclo anual de las lluvias en términos de variables climáticas de gran escala resultó un trabajo difícil. El modelo SDSM presentó algunos inconvenientes en los seis sitios de estudio para simular la precipitación. La simulación con datos del NCEP representa cierta tendencia del ciclo anual, aunque existieron sobreestimaciones y subestimaciones enero fue el mejor modelado, como se observa para la estación de Bataquez (Figura 4). En esta misma gráfica se aprecia que la modelación con el Hadley A2 y B2 fue muy deficiente pues no fue capaz de reproducir a nivel mensual el ciclo anual de lluvia. Sin embargo el valor total anual promedio fue muy próximo al observado de 1961-1990. Con base en los resultados se puede inferir que el modelo parece ser adecuado para simular los valores medios mensuales de la Tmax y Tmin, así como su variabilidad en términos de la varianza; sin embargo para la precipitación, su aplicación solo es rescatable en valores anuales totales promedio.

\section{Validación con el LARS WG}

Para la etapa de validación el LARS WG corrobora si las series generadas pueden considerarse como provenientes de la misma población. Esta comprobación que determina si los valores medios, las desviaciones estándar y las distribuciones de los datos sintéticos son perceptiblemente diferentes de los datos observados, se realiza mediante las pruebas estadísticas: t-student, $F$ de Snedecor y chi-cuadrada $\left(x^{2}\right)$ respectivamente. Estas pruebas 
estadísticas asumen que el tiempo observado es una muestra aleatoria de alguna distribución existente, la cual representa el clima verdadero en un sitio. Todas las pruebas muestran las diferencias en las distribuciones generadas a partir de los datos observados y sintéticos. Cada una de estas pruebas calcula un estadístico de prueba y un correspondiente valor $p$ de significancia, el cual indica la probabilidad de que los datos observados y generados provienen de la misma distribución, por lo tanto el valor de $p$ es usado para aceptar la hipótesis nula $\left(H_{0}\right)$ de que los dos conjuntos de datos pueden provenir de la misma población; entonces un valor de p por debajo del nivel de significancia establecido en 0.01 o 0.05 , indica que el clima simulado es poco probable que sea el mismo que el clima verdadero (Semenov et al., 2002). Un ejemplo de los datos de salida de Tmin para la estación Presa Morelos se muestra en la Tabla 1.

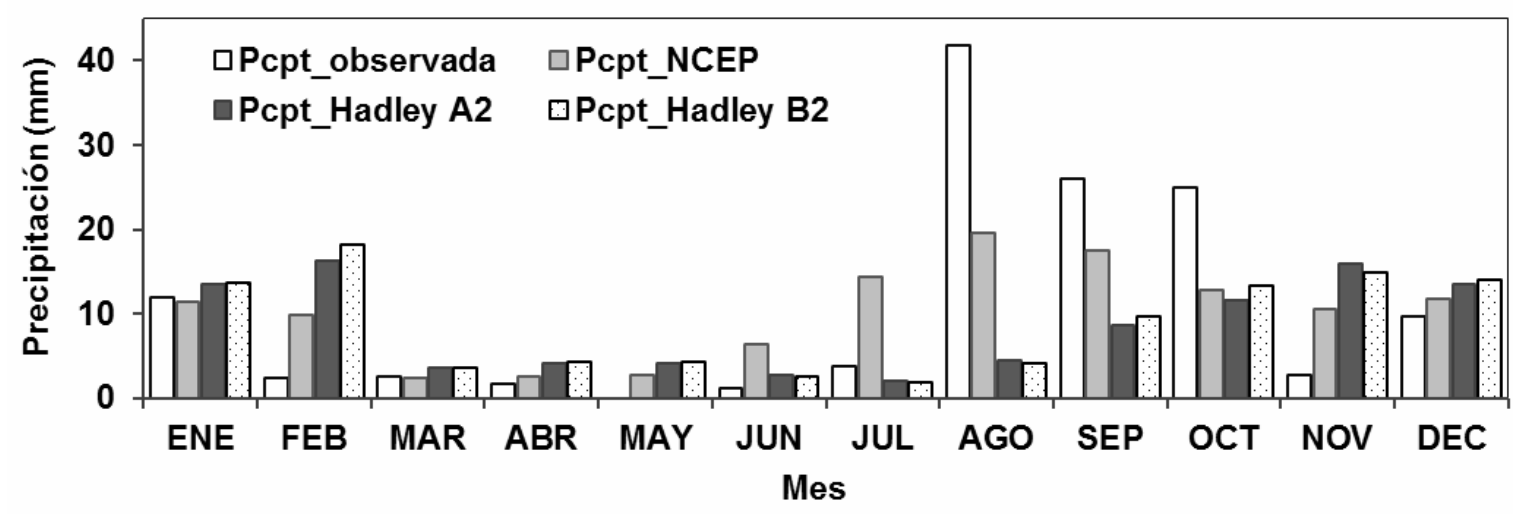

Fig. 4: Simulación de la precipitación total mensual del periodo 1961-1990 con observaciones de la estación Bataquez usando el modelo SDSM

Tabla 1: Ejemplo de los datos de salida para la validación de la temperatura mínima media de la estación Presa Morelos utilizando el modelo LARS WG

\begin{tabular}{|c|c|c|c|c|c|c|c|c|c|c|}
\hline \multirow{2}{*}{ Mes } & \multicolumn{2}{|c|}{ Observada } & \multicolumn{2}{|c|}{ Generada } & \multirow{2}{*}{$\begin{array}{c}\text { Prueba } \\
t\end{array}$} & \multirow{2}{*}{$\begin{array}{l}\text { Valor } \\
p\end{array}$} & \multirow{2}{*}{$\begin{array}{c}\text { Prueba } \\
\text { F }\end{array}$} & \multirow{2}{*}{$\begin{array}{l}\text { Valor } \\
p\end{array}$} & \multirow{2}{*}{$\begin{array}{c}\text { Prueba } \\
x^{2}\end{array}$} & \multirow{2}{*}{$\begin{array}{l}\text { Valor } \\
\mathrm{p}\end{array}$} \\
\hline & Media & $s d$ & Media & $s d$ & & & & & & \\
\hline$E$ & 5.33 & 1.548 & 5.51 & 0.607 & -0.729 & 0.468 & 6.504 & 0 & 0.106 & 0.99 \\
\hline $\mathrm{F}$ & 6.81 & 1.819 & 6.64 & 0.573 & 0.62 & 0.537 & & 0 & & 0.99 \\
\hline M & 8.99 & 1.302 & 9.15 & 0.575 & -0.744 & 0.459 & 5.127 & 0 & 0.105 & 0.99 \\
\hline$A$ & 11.89 & 1.57 & 11.97 & & & & & 0 & 0.106 & 0.99 \\
\hline M & 15.84 & 1.415 & 15.85 & 0.590 & 35 & 0.972 & & 0 & & 0.99 \\
\hline $\mathrm{J}$ & 20.17 & 1.494 & 19.89 & 0.489 & 91 & 0.237 & 34 & 0 & 0.158 & 0.91 \\
\hline $\mathrm{J}$ & 24.56 & 1.857 & 24.77 & 0.488 & 43 & 0.460 & 14. & 0 & & 0.99 \\
\hline$A$ & 24.54 & 1.605 & 24.22 & 0.544 & 1.293 & 0.200 & 8.705 & 0 & 0.210 & 0.64 \\
\hline$S$ & 20.97 & 1.829 & 20.59 & & & & & 0 & & 0.99 \\
\hline $\mathrm{O}$ & 14.83 & 1.632 & 14.93 & 0.564 & -0.391 & 0.697 & 8.373 & 0 & 0.106 & 0.99 \\
\hline $\mathrm{N}$ & 9.20 & 1.437 & 9.65 & 0.555 & -1.953 & 0.055 & 6.704 & 0 & 0.053 & 1.00 \\
\hline $\mathrm{D}$ & 5.61 & 1.33 & 5.68 & 0.558 & -0.335 & 0.739 & 5.681 & 0 & 0.106 & 0.99 \\
\hline
\end{tabular}

En la Tabla 2 se muestra el número de estaciones que no pasaron cualquiera de las tres pruebas estadísticas en algún mes. Respecto a los valores medios, los valores de $p$ de la prueba $t$ indican que para la precipitación, el generador de tiempo mostró una simulación adecuada excepto para el mes de julio para la estación Mexicali; para la temperatura mínima en la estación Rí́to no se simuló adecuadamente en los meses de enero y agosto; y finalmente para la temperatura máxima se rechazó la $\mathrm{H}_{0}$ en el mes de enero para la estación Juárez, en el mes de mayo para la estación Presa Morelos, y enero y agosto para la estación Rí́to. Respecto a la variabilidad del clima en términos de los valores $\mathrm{p}$ correspondientes a la prueba $\mathrm{F}$ que compara la desviación estándar calculada por el programa entre el clima verdadero y el clima simulado, se tiene que tanto para la 
temperatura máxima como mínima, en los seis sitios de estudio se rechazó la $\mathrm{H}_{0}$ en todos los meses; sin embargo, los resultados fueron relativamente mejores para la precipitación, siendo enero, febrero, agosto y diciembre, los meses en que se representó apropiadamente el clima en toda la región, al contrario de junio en el que la $\mathrm{H}_{0}$ se rechazó para todas las estaciones analizadas, lo cual podría no ser tan preocupante debido a que es un mes predominantemente seco. La prueba chi-cuadrada $x^{2}$ que ejecuta el generador, revela que las distribuciones de la serie observada y la serie sintética tienen la misma distribución, para la Tmin y Tmax en las seis estaciones de estudio. Para la precipitación, el caso más crítico ocurre en el mes de junio.

Tabla 2: Valores de $\mathrm{p}$ calculados de las tres pruebas estadísticas utilizadas para comparar los datos observados del periodo 1961-1990 con los datos sintéticos generados por LARS WG

\begin{tabular}{cccccccccccccc}
\hline \multirow{2}{*}{ Variable } & Prueba & \multicolumn{1}{c}{ Palor de probabilidad $(\mathrm{p})$ mensual } \\
& estadística & $\mathrm{E}$ & $\mathrm{F}$ & $\mathrm{M}$ & $\mathrm{A}$ & $\mathrm{M}$ & $\mathrm{J}$ & $\mathrm{J}$ & $\mathrm{A}$ & $\mathrm{S}$ & $\mathrm{O}$ & $\mathrm{N}$ & $\mathrm{D}$ \\
\hline $\mathrm{P}$ & $\mathrm{t}$ & 0 & 0 & 0 & 0 & 0 & 1 & 0 & 0 & 0 & 0 & 0 & 0 \\
& $\mathrm{~F}$ & 0 & 0 & 1 & 1 & 4 & 6 & 1 & 0 & 1 & 1 & 2 & 0 \\
& $\mathrm{X}^{2}$ & 0 & 0 & 0 & 1 & 4 & 5 & 0 & 0 & 0 & 0 & 0 & 0 \\
\hline \multirow{2}{*}{ Tmin } & $\mathrm{t}$ & 1 & 0 & 0 & 0 & 0 & 0 & 0 & 1 & 0 & 0 & 0 & 0 \\
& $\mathrm{~F}$ & 6 & 6 & 6 & 6 & 6 & 6 & 6 & 6 & 6 & 6 & 6 & 6 \\
& $\mathrm{X}^{2}$ & 0 & 0 & 0 & 0 & 0 & 0 & 0 & 0 & 0 & 0 & 0 & 0 \\
\hline \multirow{2}{*}{$\operatorname{mmax}$} & $\mathrm{t}$ & 2 & 0 & 0 & 0 & 1 & 0 & 0 & 1 & 0 & 0 & 0 & 0 \\
& $\mathrm{~F}$ & 6 & 6 & 6 & 6 & 6 & 6 & 6 & 6 & 6 & 6 & 6 & 6 \\
& $\mathrm{X}^{2}$ & 0 & 0 & 0 & 0 & 0 & 0 & 0 & 0 & 0 & 0 & 0 & 0 \\
\hline
\end{tabular}

Con base en el análisis de los resultados obtenidos en las pruebas estadísticas, se puede observar para la región del Valle de Mexicali que el modelo planteado tiene la habilidad de reproducir la temperatura máxima y mínima adecuadamente en términos de valores medios, sin embargo, no muestra una habilidad adecuada para la simulación de la variabilidad climática en términos de desviación estándar. Para la precipitación mostró un buen ajuste para los valores medios y una deficiencia en cuanto a la variabilidad climática para los meses de mayo y junio, lo cual en un momento dado podría no ser tan importante, ya que estos meses son los más secos del ciclo anual de lluvias. Aunque el LARS WG es un generador que ha demostrado ser un buen simulador para varios climas (Semenov et al., 1998), es de esperarse que en la región bajo estudio, el clima no puede ser aproximado a la distribución teórica que propone el generador, pues es poco probable que esta sea capaz de coincidir con la gran variedad de climas en el mundo.

\section{Generación de escenarios de cambio climático}

Se generaron los escenarios de cambio climático obteniendo dos climatologías, la 2020 (periodo 2010-2039) y 2050 (periodo 2040-2069). Los resultados muestran que las tendencias para Tmax, Tmin y P (Tabla 3, 4 y 5), son coherentes entre las estaciones de estudio. Se calculó un promedio espacial de las seis estaciones de estudio de la región del Valle de Mexicali observando que cuando los cambios se derivan del MCG HadCM3 aplicando el SDSM, se obtiene una tendencia al aumento en los valores promedio anuales para Tmin y Tmax bajo el escenario A2 de $0.8{ }^{\circ} \mathrm{C}$ y 1.6 ${ }^{0} \mathrm{C}$ para la climatología 2020 y 2050 respectivamente, en tanto que para el escenario B2 son de $1.0^{\circ} \mathrm{C}$ y $1.6^{\circ} \mathrm{C}$ para las mismas climatologías consideradas. Para la precipitación la anomalía es positiva y semejante entre el escenario A2 y B2, encontrando un aumento aproximado del $20 \%$ para la climatología 2020 y del $50 \%$ para la climatología 2050.

Aplicando el esquema LARS WG a partir del MM, se encontró que para el escenario A2 en el periodo 2010-2039 se observa un incremento de Tmin de $0.9^{\circ} \mathrm{C}$, mientras que para el periodo 2040-2069 de $1.6{ }^{\circ} \mathrm{C}$. En cuanto a Tmax se tiene un incremento de $1.0{ }^{\circ} \mathrm{C}$ y $1.6{ }^{\circ} \mathrm{C}$, para ambos periodos respectivamente. El escenario A1B muestra incrementos para Tmin y Tmax de $1.0{ }^{\circ} \mathrm{C}$ para el periodo $2010-2039$ y de $1.8{ }^{\circ} \mathrm{C}$ para el periodo $2040-2069$. Respecto a la precipitación, los cambios son anomalías negativas mayormente acentuadas para el escenario $\mathrm{A} 2$ de $-15.8 \%$ para 
la climatología 2020 y de $-20.4 \%$ para la climatología 2050, mientras que para el escenarios A1B, es de $-5.0 \%$ y $-10.69 \%$, para estas mismas climatologías respectivas.

Tabla 3: Anomalías de temperatura máxima absoluta obtenidas bajo cambio climático para la región del Valle de Mexicali, aplicando dos técnicas de reducción a escala

\begin{tabular}{|c|c|c|c|c|c|c|c|c|c|}
\hline \multirow{2}{*}{$\begin{array}{l}\text { Técnica de } \\
\text { reducción } \\
\text { de escala }\end{array}$} & \multirow[t]{2}{*}{ MCG } & \multirow[t]{2}{*}{ Escenario } & \multirow[t]{2}{*}{ Periodo } & \multicolumn{6}{|c|}{$\begin{array}{l}\text { Anomalía media anual de temperatura } \\
\text { máxima (Tmax) en diferencia absoluta }\left({ }^{\circ} \mathrm{C}\right.\end{array}$} \\
\hline & & & & 2003 & 2009 & 2011 & 2033 & 2037 & 26076 \\
\hline \multirow{4}{*}{ LARS WG } & \multirow{4}{*}{ Multiensamble } & & 2010-2039 & 0.9 & 1.0 & 0.9 & 1.0 & 1.0 & 1.0 \\
\hline & & & 2040-2069 & 1.6 & 1.6 & 1.6 & 1.6 & 1.6 & 1.6 \\
\hline & & \multirow{2}{*}{ A1B } & 2010-2039 & 1.0 & 1.1 & 1.0 & 1.0 & 1.0 & 1.0 \\
\hline & & & 2040-2069 & 1.8 & 1.8 & 1.8 & 1.7 & 1.7 & 1.8 \\
\hline \multirow{4}{*}{ SDSM } & \multirow{4}{*}{ HADCM3 } & \multirow{2}{*}{ A2 } & 2010-2039 & 0.5 & 0.7 & 0.9 & 0.9 & 0.9 & 0.8 \\
\hline & & & 2040-2069 & 1.1 & 1.4 & 1.8 & 1.9 & 1.9 & 1.7 \\
\hline & & \multirow{2}{*}{ B2 } & 2010-2039 & 0.7 & 0.9 & 1.1 & 1.2 & 1.1 & 1.0 \\
\hline & & & $2040-2069$ & 1.1 & 1.4 & 1.7 & 1.8 & 1.8 & 1.6 \\
\hline
\end{tabular}

Tabla 4: Anomalías de temperatura mínima en diferencia absoluta $\left({ }^{\circ} \mathrm{C}\right)$ obtenidas bajo cambio climático para la región del Valle de Mexicali, aplicando dos técnicas de reducción a escala

\begin{tabular}{|c|c|c|c|c|c|c|c|c|c|}
\hline \multirow{2}{*}{$\begin{array}{l}\text { Técnica de } \\
\text { reducción } \\
\text { de escala }\end{array}$} & \multirow[t]{2}{*}{ MCG } & \multirow[t]{2}{*}{ Escenario } & \multirow[t]{2}{*}{ Periodo } & \multicolumn{6}{|c|}{$\begin{array}{l}\text { Anomalía media anual de temperatura } \\
\text { mínima (Tmin) en diferencia absoluta }\left({ }^{\circ} \mathrm{C}\right)\end{array}$} \\
\hline & & & & 2003 & 2009 & 2011 & 2033 & 2037 & 26076 \\
\hline \multirow{4}{*}{ LARS WG } & \multirow{4}{*}{ Multiensamble } & \multirow{2}{*}{$\mathrm{A} 2$} & 2010-2039 & 0.9 & 0.9 & 0.9 & 0.9 & 1 & 0.8 \\
\hline & & & $2040-2069$ & 1.6 & 1.6 & 1.6 & 1.6 & 1.6 & 1.6 \\
\hline & & \multirow{2}{*}{ A1B } & 2010-2039 & 1.0 & 1.0 & 1.0 & 1.0 & 1.0 & 1.0 \\
\hline & & & 2040-2069 & 1.8 & 1.8 & 1.8 & 1.7 & 1.7 & 1.8 \\
\hline \multirow{4}{*}{ SDSM } & \multirow{4}{*}{ HADCM3 } & \multirow{2}{*}{ A2 } & $2010-2039$ & 0.6 & 0.7 & 0.8 & 1.0 & 0.9 & 0.9 \\
\hline & & & $2040-2069$ & 1.2 & 1.4 & 1.6 & 2.0 & 1.7 & 1.9 \\
\hline & & \multirow{2}{*}{$\mathrm{B} 2$} & $2010-2039$ & 0.7 & 0.9 & 1.0 & 1.1 & 1.0 & 1.0 \\
\hline & & & 2040-2069 & 1.2 & 1.4 & 1.6 & 2.0 & 1.7 & 1.8 \\
\hline
\end{tabular}

Tabla 5: Anomalías de precipitación en diferencia porcentual (\%) obtenidas bajo cambio climático para la región del Valle de Mexicali, aplicando dos técnicas de reducción a escala

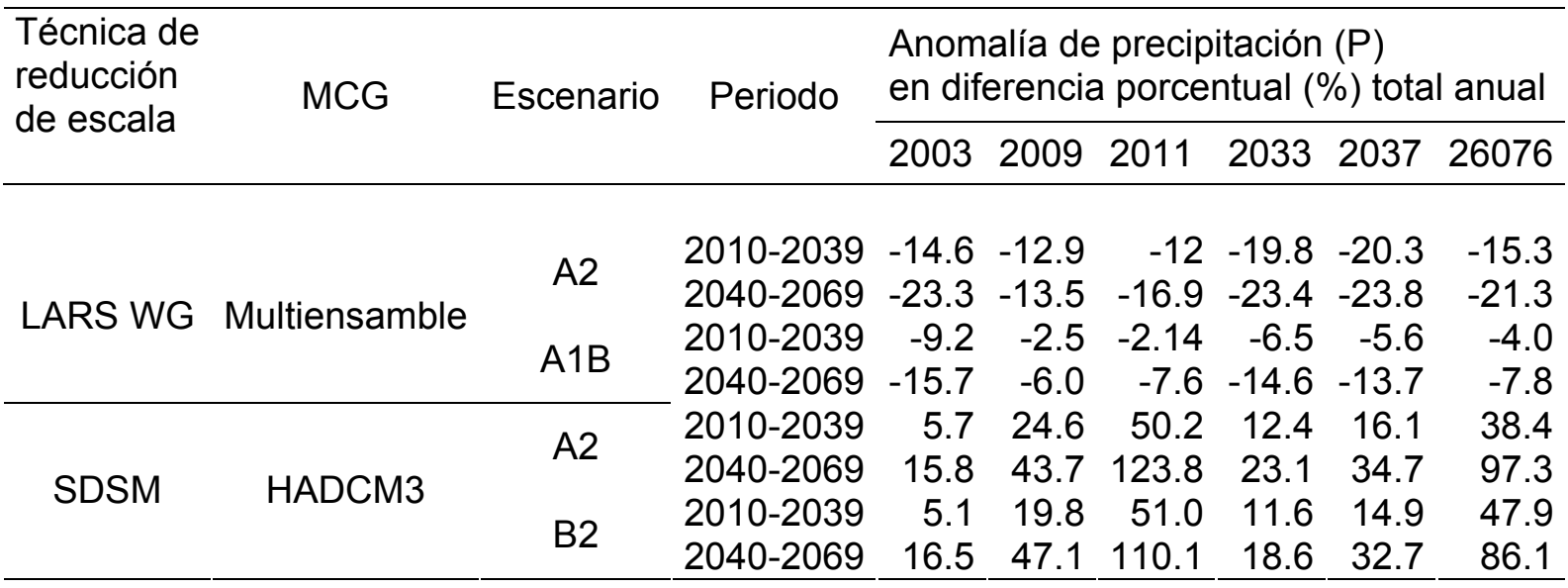

Del análisis anterior es posible observar que para temperatura, respecto al escenario A2, el SDSM registra un mayor calentamiento en el escenario B2 en la climatología 2020 y el LARS WG en el escenario A1B en la climatología 2050. Ambas diferencias son del orden de $0.2{ }^{\circ} \mathrm{C}$. Esta diferencia se explica entendiendo que el escenario A2 corresponde a una evolución de emisiones de GEl más creciente que el $\mathrm{A} 1 \mathrm{~B}$ y el B2, la cual es evidente para finales del presente siglo, sin embargo 
los horizontes analizados (2010-2039 y 2040-2069) pueden corresponder a un periodo de transición que caracteriza al escenario A1B por una orientación más mundial con una mayor demanda de recursos y servicios, y el escenario B2 por cambios lentos a tecnologías no fósiles.

\section{Escenario comparativo A2 entre el SDSM y el LARS WG}

El escenario comparativo entre el SDSM y el LARS WG fue el escenario A2. En términos de temperatura media anual ambas técnicas de escalamiento mostraron anomalías positivas semejantes, por un lado con el SDSM se determina para la Tmax y la Tmin un aumento de $0.8^{\circ} \mathrm{C}$ y $1.6{ }^{\circ} \mathrm{C}$ para la climatología 2020 y 2050 respectivamente. Por otro lado el LARS WG muestra para la climatología 2020 un aumento de Tmax de $1.0^{\circ} \mathrm{C}$ y para la Tmin de $0.9^{\circ} \mathrm{C}$; para la climatología 2050 tanto para la Tmax como para la Tmin estima un aumento de $1.6{ }^{\circ} \mathrm{C}$. A nivel anual la diferencia entre el LARS WG y el SDSM, para el escenario y horizontes comparados, es de $0.2{ }^{\circ} \mathrm{C}$ para la Tmax y de $0.1{ }^{\circ} \mathrm{C}$ para Tmin para la climatología 2020 y no se mostró diferencia para la climatología 2050, lo cual indica que el aumento de la temperatura tendrá una tendencia lineal. En términos de cambio porcentual en la precipitación anual total, el HadCM3 estima aumentos con el SDSM de $24.60 \%$ para la climatología 2020, a $56.4 \%$ para la climatología 2050; por el contrario, LARS WG proyecta disminución en el total del ciclo anual de lluvia utilizando el MM de $-15.81 \%$ para la climatología 2020 y -20.3\% para la climatología 2050.

\section{Escenarios y tendencias}

De acuerdo a las anomalías proyectadas para la región del Valle de Mexicali, los cambios esperados de aumentos para temperatura y disminuciones para precipitación están acordes a las tendencias de los últimos 100 años en el trabajo que Magaña describe en INE (2009), por un lado, conforme al análisis de temperatura de superficie, indica que en la mayor parte de México han ocurrido aumentos que varían de región en región. Los cambios observados en temperatura media anual varían de $-0.5^{\circ} \mathrm{C}$, en ciertas partes del noreste, a cerca de $1.5^{\circ} \mathrm{C}$, en el noroeste. Por otra parte, muestra que las tendencias de la precipitación para México sugieren una especie de dipolo: incremento en el sur y disminución en el norte. Según estas tendencias, para nuestra zona de estudio son claramente positivas para la temperatura $\left(0.6-0.8{ }^{\circ} \mathrm{C}\right)$ y negativas para la precipitación (0 a $-3 \%$ \%). Lo anterior coincide también con SEMARNAT-INE (2009) en un calentamiento acelerado de $0.7^{\circ} \mathrm{C}$ para el territorio mexicano en el periodo 1999-2008 y de acuerdo la distribución de lluvia de 1941-2008, la precipitación promedio anual de la región de estudio fue la más baja en todo el país.

\section{CONCLUSIONES}

EI LARS WG y el SDSM son dos técnicas de reducción de escala estructuralmente diferentes y ambas resultaron computacionalmente económicas y relativamente fáciles de aplicar, siendo el SDSM más demandante en tiempo y conocimiento especializado.

EI LARS WG reproduce de forma adecuada los valores medios mensuales de temperatura máxima, temperatura mínima y precipitación. Se encontró una escasa habilidad del modelo para reproducir la oscilación térmica anual.

EI SDSM mostró eficiencia para simular el comportamiento de la temperatura y su variabilidad; y por el contrario, una pobre simulación para la precipitación en ambas características.

Ambas técnicas estiman un aumento de temperatura para la climatología 2020 y 2050 , obteniendo diferencias poco significativas a nivel promedio regional en relación al escenario comparado A2. Para precipitación, el LARS WG estima disminuciones y el SDSM proyecta aumentos, por lo que será necesaria para esta variable la evaluación de otras estrategias de reducción de escala.

\section{REFERENCIAS}

CCIS, Canadian Climate Impacts Scenarios, (en línea)

http://www.cics.uvic.ca/scenarios/sdsm/select.cgi. Acceso: 6 abril (2010). 
Estrada F., V. Guerrero y C. Gay, Una nueva metodología para realizar downscaling estadístico y para la estimación de impactos potenciales de cambio climático en el consumo de energía en las ciudades. VII Congreso de la Asociación Española de Climatología. Clima, Ciudad y Ecosistemas, 73-82, España, Madrid 27 a 29 Noviembre (2010).

García, E., Modificaciones al sistema de clasificación climática de Köppen, 2a edición, 7-56, Instituto de Geografía, UNAM, México, D. F. (1973).

INE, Instituto Nacional de Ecología, Adaptación a los impactos del cambio climático en los humedales costeros del Golfo de México, $1^{\text {a }}$ edición, Vol. II, 571-588, Buenfil Ed., México, D. F. (2009).

Kalnay, E., M. Kanamitsu y R. Kistler, The NCEP/NCAR 40-Year Reanalysis Project, Bolletin American Meteorological Society, 77, 437-471 (1996).

Magaña, O.V., Guía para Generar y Aplicar Escenarios Probabilísticos Regionales de Cambio Climático en la Toma de Decisiones, Instituto Nacional de Ecología, 2010 (en línea) http://zimbra.ine.gob.mx/escenarios. Acceso: 20 abril (2010).

Nakicenovic N. y otros 27 autores, Special Report on Emissions Scenarios: A Special Report of Working Group III of the Intergovernmental Panel on Climate Change, Cambridge University Press. Cambridge (2000).

Raynal, J. A. y M. E. Raynal, Cálculo de los límites de confianza de la Distribución de Probabilidad de valores extremos tipo i para dos poblaciones, Información Tecnológica, ISSN: 0718-0764 (en línea), 15(1), 2004. http:www.scielo.cl/scielo.php. Acceso: 10 de noviembre (2011).

SEMARNAT-INE, Secretaria de Medio Ambiente y Recursos Naturales-Instituto Nacional de Ecología, México Cuarta Comunicación Nacional ante la Convención Marco de las Naciones Unidas sobre el Cambio Climático, México, D. F. (2009).

Semenov M. A. y P. Stratonovitch, Use of multi-model ensembles from global climate models for assessment of climate change impacts, Clim. Res., 41, 1-14 (2010).

Semenov, M. A. y E. M. Barrow, LARS-WG a Stochastic Weather Generator for Use in Climate Impact Studies. User Manual, 2002 (en línea) http://www.rothamsted.bbsrc.ac.uk/masmodels/download/LARS-WG-Manual.pdf. Acceso: 5 abril (2010).

Semenov, M. A., Development of high-resolution UKCIP02-based climate change scenarios in the UK, Agric. For Meteorol., 144, 127-138 (2007).

Semenov, M. A., R. J. Brooks, E. M. Barrow y C. W. Richardson, Comparison of the WGEN and LARS-WG stochastic weather generators in diverse climates, Clim. Res., 10, 95-107 (1998).

Solomon S. y otros siete autores, IPCC-WGI Climate Change 2007: The Physical Science Basis. Contribution of Working Group I to the Fourth Assessment Report of the Intergovernmental Panel on Climate Change, Cambridge University Press, Cambridge, United Kingdom and New York, NY, USA (2007).

Wilby, R. L. y C.W. Dawson, SDSM 4.2 -A decision support tool for the assessment of regional climate change impacts. User Manual, 2007 (en línea) https://copublic.lboro.ac.uk/cocwd/SDSM/index.html. Acceso: 30 de abril (2010).

Zermeño, D., Análisis probabilístico de escenarios escalados de precipitación y temperatura bajo cambio climático en México, tesis de Maestría en Ciencias, Centro de Ciencias de la Atmósfera, UNAM, México (2008). 